\title{
Supporting Information for \\ Crystal Structure and Stability of Ammonium Azide under High Pressure
}

Guozhao Zhang ${ }^{1,2}$, Haiwa Zhang ${ }^{2}$, Sandra Ninet ${ }^{2}$, Hongyang Zhu ${ }^{3}$, Cailong Liu ${ }^{1,4}$, Jean-

Paul Itié $e^{5}$ Chunxiao Gao ${ }^{l, *}$, and Frédéric Datchi ${ }^{2}$ *

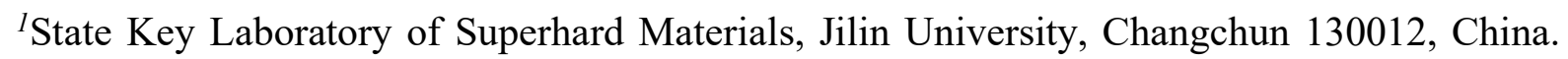

${ }^{2}$ Institut de Minéralogie, de Physique des Matériaux et de Cosmochimie (IMPMC), Sorbonne Université, CNRS UMR 7590, MNHN, 4 Place Jussieu, F-75005 Paris, France.

${ }^{3}$ School of Physics and Electronic Engineering, Linyi University, Linyi 276005, China

${ }^{4}$ Shandong Key Laboratory of Optical Communication Science and Technology, School of Physical Science and Information Technology of Liaocheng University, Liaocheng 252059, China

${ }^{5}$ Synchrotron Soleil, L'Orme des Merisiers, Saint-Aubin, BP 48, 91192 Gif-sur-Yvette Cedex, France.

*Corresponding Authors e-mail: cc060109@qq.com; frederic.datchi@sorbonne-universite.fr

Figure S1. Raman spectra of the synthesized $\mathrm{NH}_{4} \mathrm{~N}_{3}$ powder sample at ambient conditions.

Figure S2. X-ray diffraction image of the AA sample embedded in nitrogen pressure medium at $28.2 \mathrm{GPa}$ and $300 \mathrm{~K}$

Figure S3. Calculated Raman spectra of AA- $P 2 / c$ at different pressures.

Figure S4. Comparison of the experimental Raman frequencies of AA as a function of pressure for the sample compressed in argon pressure medium and for the one with no pressure medium.

Table S1. Comparison of our calculation results for the DFT optimized structures at ambient pressure with previous theoretical calculations.

Table S2. Details of the Rietveld refinement of the XRD patterns at different pressures.

Table S3. Lattice parameters and volume of the $P 2 / c$ phase as a function of pressure obtained in present theoretical calculations.

Table S4. Calculated optical modes of AA-P2/c at 6.0 GPa. 


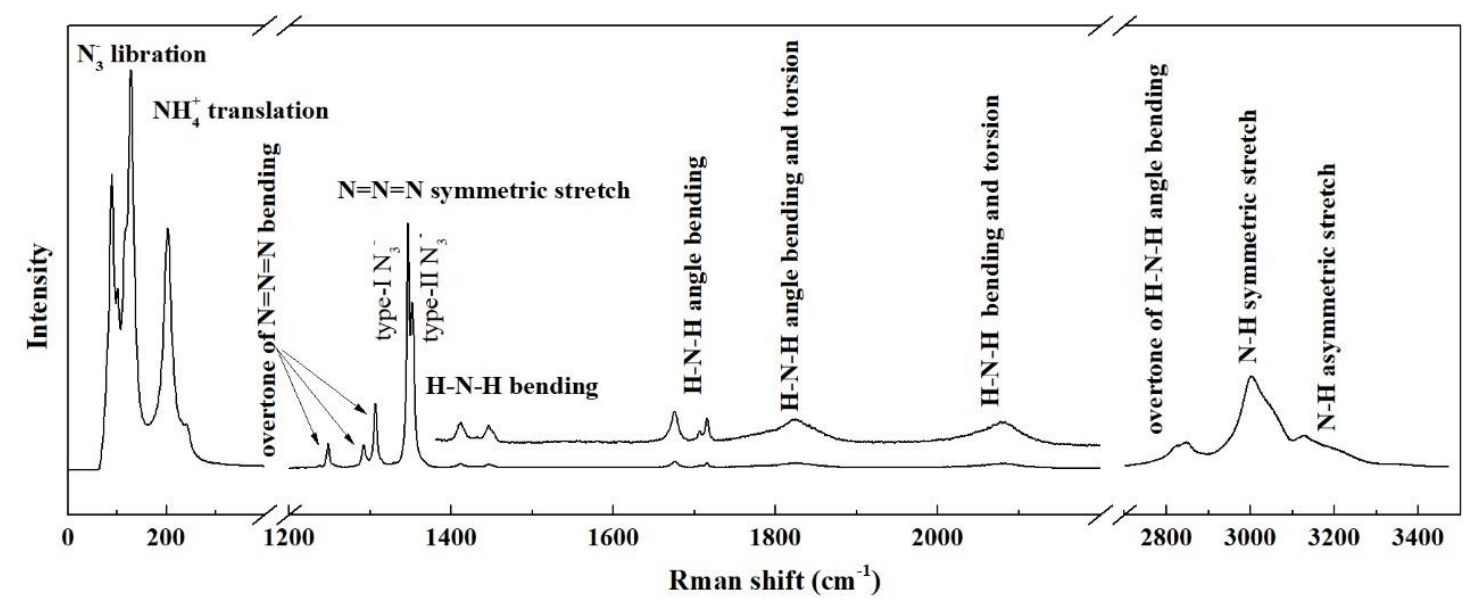

Figure S1. Raman spectra of the synthesized $\mathrm{NH}_{4} \mathrm{~N}_{3}$ powder sample at ambient conditions. 


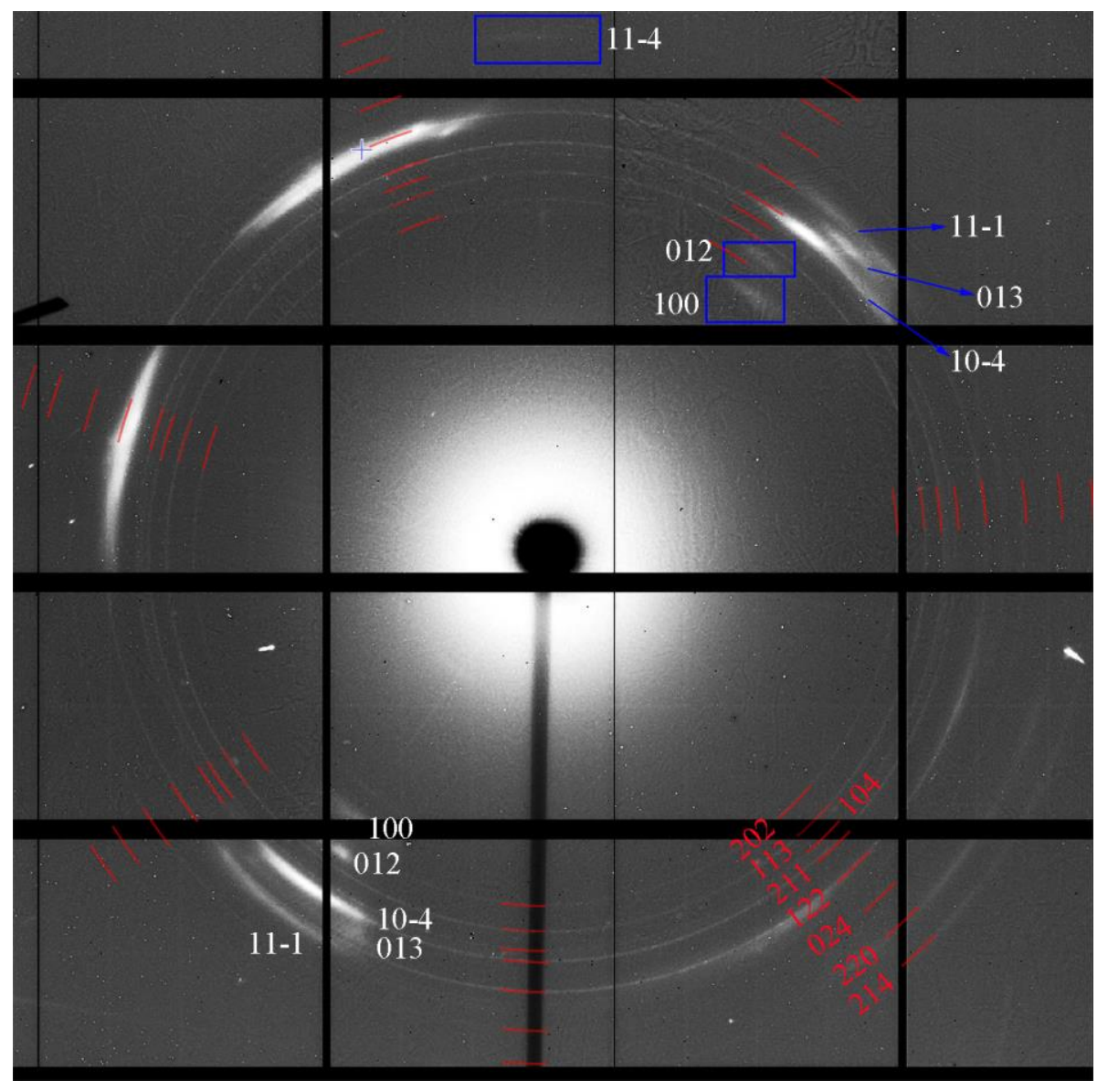

Figure S2. X-ray diffraction image of the AA sample embedded in nitrogen pressure medium at $28.2 \mathrm{GPa}-300 \mathrm{~K}$, showing that this sample is highly textured. The (hkl) indices in white refer to the reflections from the AA-P2/c phase. The red dashed circles with red (hkl) indices represent the diffraction rings from the $\varepsilon$ phase of nitrogen ${ }^{1}$. 


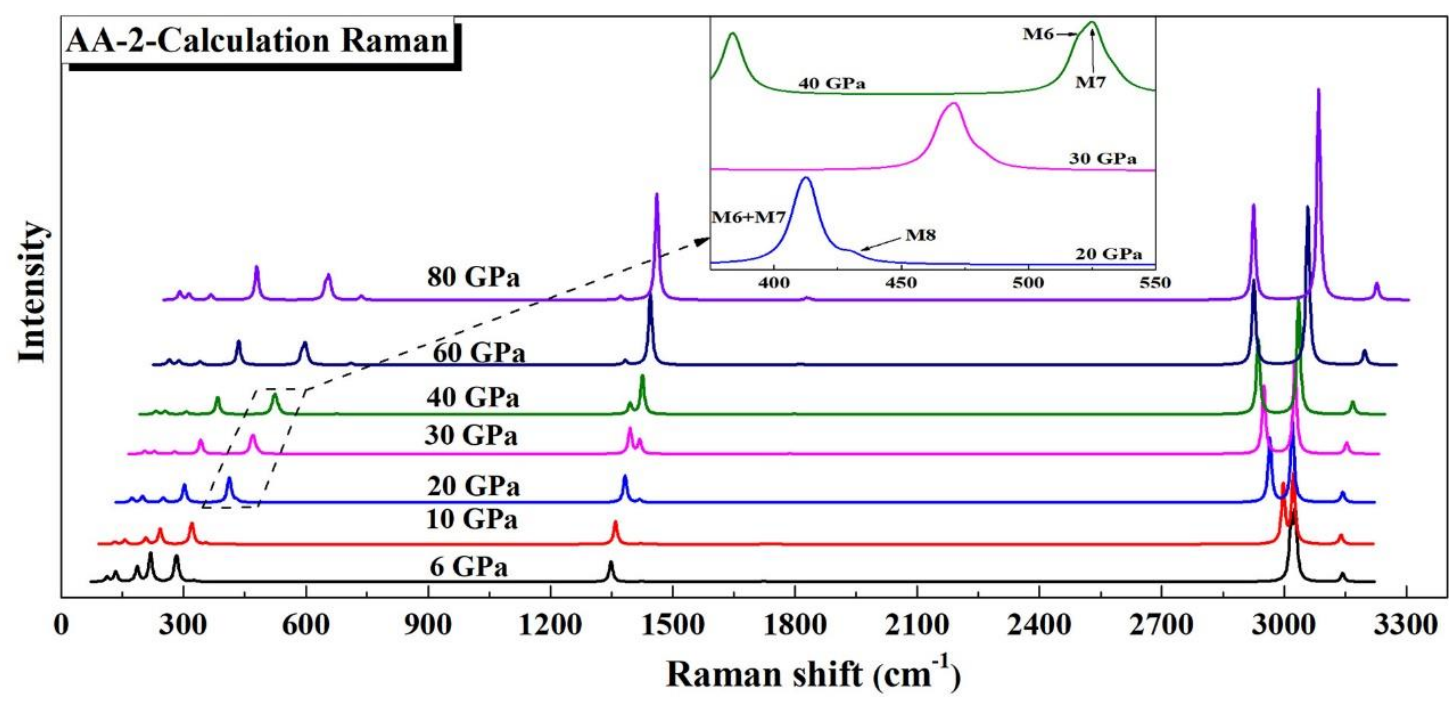

Figure S3. Calculated Raman spectra of AA- $P 2 / c$ at different pressures.
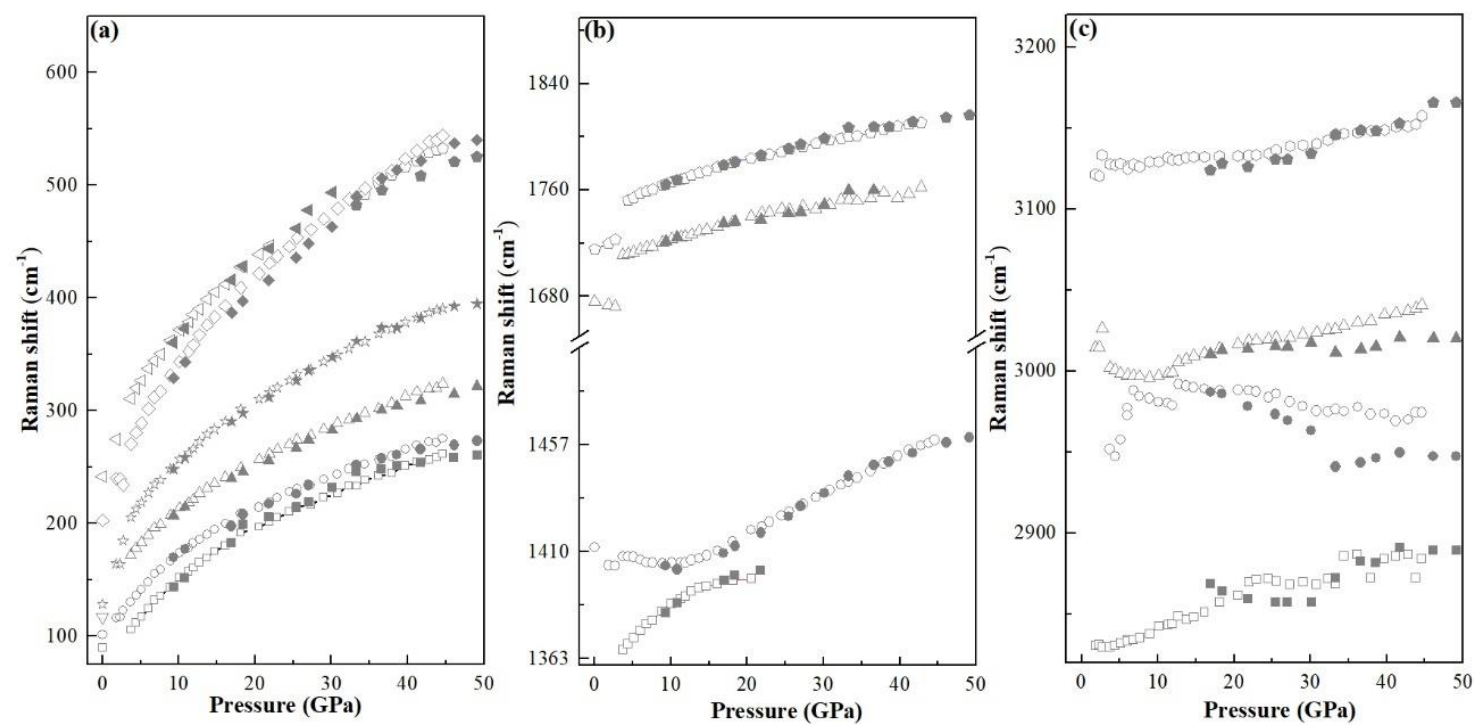

Figure S4. Comparison between the experimental Raman frequencies of AA as a function of pressure, measured with the sample with argon pressure medium (open symbols) and no pressure medium (solid symbols). The difference in frequency measured in the two runs is small for most of the modes, which results from the fact that AA is a soft material and act itself as a good pressure medium. The larger difference observed in the modes near $3000 \mathrm{~cm}^{-1}$ comes from the fact that this band is composed of three modes which are not well resolved, inducing larger uncertainties in the postion of individual components. 
Table S1. Comparison of our calculation results for the DFT optimized structures at ambient pressure with previous theoretical calculations.

\begin{tabular}{|c|c|c|c|}
\hline Phase & Parameter & This work & Other calculations \\
\hline \multirow{5}{*}{$\begin{array}{l}\text { AA-Pmna } \\
(\mathrm{Z}=4)\end{array}$} & $\mathrm{a}(\AA)$ & 9.010 & $9.094^{\mathrm{a}}, 9.205^{\mathrm{b}}, 8.754^{\mathrm{c}}, 9.015^{\mathrm{d}}$ \\
\hline & $\mathrm{b}(\AA)$ & 3.887 & $3.904^{\mathrm{a}}, 3.809^{\mathrm{b}}, 3.636^{\mathrm{c}}, 3.809^{\mathrm{d}}$ \\
\hline & $\mathrm{c}(\AA)$ & 8.620 & $8.627^{\mathrm{a}}, 8.546^{\mathrm{b}}, 8.356^{\mathrm{c}}, 8.551^{\mathrm{d}}$ \\
\hline & $\mathrm{V}\left(A^{3}\right)$ & 304.88 & $306.28^{\mathrm{a}}, 299.6^{\mathrm{b}}, 266.0^{\mathrm{c}}, 293.7^{\mathrm{d}}$ \\
\hline & $\mathrm{E}_{0}(\mathrm{eV} /$ atom $)$ & -146.267 & $-143.512^{\mathrm{c}},-143.51^{\mathrm{d}}$ \\
\hline \multirow{6}{*}{$\begin{array}{l}\mathrm{AA}-P 2 / \mathrm{c} \\
(\mathrm{Z}=2)\end{array}$} & $\mathrm{a}(\AA)$ & 3.669 & $2.816^{\mathrm{e}}$ \\
\hline & $\mathrm{b}(\AA)$ & 3.665 & $2.859^{\mathrm{e}}$ \\
\hline & $\mathrm{c}(\AA)$ & 13.481 & $10.112^{\mathrm{e}}$ \\
\hline & $\beta\left(^{\circ}\right)$ & 123.32 & $118.08^{\mathrm{e}}$ \\
\hline & $\mathrm{V}\left(A^{3}\right)$ & 151.44 & $71.83^{\mathrm{e}}$ \\
\hline & $\mathrm{E}_{0}(\mathrm{eV} / \mathrm{atom})$ & -146.25 & \\
\hline \multirow{8}{*}{$\begin{array}{l}\mathrm{TTZ} \\
(P-1, \mathrm{Z}=4)\end{array}$} & $\mathrm{a}(\AA)$ & 10.527 & $10.575^{b}, 10.469 d$ \\
\hline & $\mathrm{b}(\AA)$ & 7.056 & $6.863^{\mathrm{b}}, 6.888^{\mathrm{d}}$ \\
\hline & $\mathrm{c}(A)$ & 4.107 & $4.026^{\mathrm{b}}, 4.081^{\mathrm{d}}$ \\
\hline & $\alpha\left(^{\circ}\right)$ & 103.52 & $102.60^{b}, 102.63^{d}$ \\
\hline & $\beta\left(^{\circ}\right)$ & 88.34 & $87.463^{\mathrm{b}}, 97.94^{\mathrm{d}}$ \\
\hline & $\gamma\left(\left(^{\circ}\right)\right.$ & 104.54 & $104.25^{\mathrm{b}}, 104.22^{\mathrm{d}}$ \\
\hline & $\mathrm{V}\left(A^{3}\right)$ & 286.96 & $278.29^{\mathrm{d}}$ \\
\hline & $\mathrm{E}_{0}(\mathrm{eV} /$ atom $)$ & -146.12 & $-143.39^{b},-143.39^{d}$ \\
\hline \multirow{6}{*}{$\begin{array}{l}\text { HNS-1 } \\
\left(P 2_{1} / m, \mathrm{Z}=2\right)\end{array}$} & $\mathrm{a}(\AA)$ & 8.452 & $8.185^{\mathrm{b}}, 8.395^{\mathrm{d}}, 8.594^{\mathrm{f}}$ \\
\hline & $\mathrm{b}(\AA)$ & 2.318 & $2.311^{\mathrm{b}}, 2.312^{\mathrm{d}}, 2.317^{\mathrm{f}}$ \\
\hline & $\mathrm{c}(\AA)$ & 3.056 & $3.064^{\mathrm{b}}, 3.05^{\mathrm{d}}, 3.002^{\mathrm{f}}$ \\
\hline & $\beta\left(^{\circ}\right)$ & 106.08 & $107.38^{\mathrm{b}}, 107.34^{\mathrm{d}}, 105.74^{\mathrm{f}}$ \\
\hline & $\mathrm{V}\left(A^{3}\right)$ & 46.56 & $55.310^{\mathrm{b}}, 56.53^{\mathrm{d}}, 57.54^{\mathrm{f}}$ \\
\hline & $\mathrm{E}_{0}($ eV/atom $)$ & -145.90 & $-143.16^{\mathrm{b}},-143.19^{\mathrm{d}}$ \\
\hline \multirow{4}{*}{$\begin{array}{l}\mathrm{HNS}-2 \\
\left(P 4_{2} / n, \mathrm{Z}=4\right)\end{array}$} & $\mathrm{a}(\AA)$ & 8.480 & $8.183^{\mathrm{d}}, 8.235^{\mathrm{g}}$ \\
\hline & $\mathrm{c}(\AA)$ & 4.684 & $4.211^{\mathrm{d}}, 4.134^{\mathrm{g}}$ \\
\hline & $\mathrm{V}\left(A^{3}\right)$ & 336.81 & $281.96^{\mathrm{d}}$ \\
\hline & $\mathrm{E}_{0}(\mathrm{eV} / \mathrm{atom})$ & -145.89 & $-143.16^{\mathrm{d}},-143.19^{\mathrm{g}}$ \\
\hline
\end{tabular}

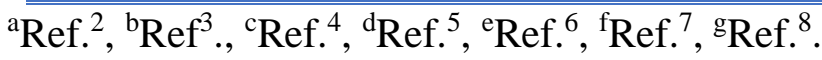


Table S2. Details of the Rietveld refinement of the XRD patterns at different pressures.

\begin{tabular}{|c|c|c|c|c|}
\hline Pressure & $0.9 \mathrm{GPa}$ & $3.0 \mathrm{GPa}$ & $7.3 \mathrm{GPa}$ & $13.8 \mathrm{GPa}$ \\
\hline cryst syst & Orthorhombic & Monoclinic & Monoclinic & Monoclinic \\
\hline space group & Pmna & $P 2 / c$ & $P 2 / c$ & $P 2 / c$ \\
\hline $\begin{array}{l}\mathrm{a}(\AA) \\
\mathrm{b}(\AA) \\
\mathrm{c}(\AA) \\
\end{array}$ & $\begin{array}{l}8.843 \\
3.715 \\
8.611 \\
\end{array}$ & $\begin{array}{l}3.532 \\
3.504 \\
11.586 \\
\end{array}$ & $\begin{array}{l}3.421 \\
3.420 \\
11.124 \\
\end{array}$ & $\begin{array}{l}3.213 \\
3.300 \\
10.464 \\
\end{array}$ \\
\hline $\begin{array}{l}\alpha(\mathrm{deg}) \\
\beta(\mathrm{deg}) \\
\gamma(\mathrm{deg})\end{array}$ & $\begin{array}{l}90 \\
90 \\
90 \\
\end{array}$ & $\begin{array}{l}90 \\
117.8 \\
90 \\
\end{array}$ & $\begin{array}{l}90 \\
116.1 \\
90 \\
\end{array}$ & $\begin{array}{l}90 \\
112.0 \\
90 \\
\end{array}$ \\
\hline $\begin{array}{l}\text { Volume } \\
(\AA 3)\end{array}$ & 282.9 & 126.9 & 116.8 & 102.9 \\
\hline R-factors & $\begin{array}{l}\mathrm{R}_{\text {Bragg }}=0.06 \\
\mathrm{R}_{\mathrm{f}}=0.076 \\
\mathrm{R}_{\mathrm{p}}=0.005 \\
\mathrm{R}_{\mathrm{wp}}=0.008 \\
\mathrm{R}_{\mathrm{exp}}=0.030 \\
\text { Chi2 }=0.07\end{array}$ & $\begin{array}{l}\mathrm{R}_{\text {Bragg }}=0.052 \\
\mathrm{R}_{\mathrm{f}}=0.105 \\
\mathrm{R}_{\mathrm{p}}=0.002 \\
\mathrm{R}_{\mathrm{wp}}=0.003 \\
\mathrm{R}_{\text {exp }}=0.029 \\
\text { Chi2 }=0.01\end{array}$ & $\begin{array}{l}R_{\text {Bragg }}=0.045 \\
R_{f}=0.077 \\
R_{p}=0.0024 \\
R_{w p}=0.0037 \\
R_{\text {exp }}=0.029 \\
\text { Chi2 }=0.016\end{array}$ & $\begin{array}{l}\mathrm{R}_{\text {Bragg }}=0.015 \\
\mathrm{R}_{\mathrm{f}}=0.016 \\
\mathrm{R}_{\mathrm{p}}=0.0025 \\
\mathrm{R}_{\mathrm{wp}}=0.004 \\
\mathrm{R}_{\text {exp }}=0.029 \\
\text { Chi2 }=0.02\end{array}$ \\
\hline Radiation & \multicolumn{4}{|c|}{$\operatorname{Mo~K\alpha }\left(\lambda_{\mathrm{K} \alpha 1}=0.70926 \AA, \lambda_{\mathrm{K} \alpha 2}=0.713590 \AA\right)$} \\
\hline $2 \theta$ range & \multicolumn{4}{|c|}{$6.0-38^{\circ}$} \\
\hline Step & \multicolumn{4}{|l|}{0.011} \\
\hline
\end{tabular}

Table S3. Experimental P-V dataset for the P2/c phase from the XRD experiments. Run 1 used a sample without pressure medium, Run 2 used nitrogen as pressure medium. Pressure in GPa, Volume in $\AA^{3} /$ molecule.

\begin{tabular}{llllll}
\hline XRD Run & Pressure & Volume & XRD Run & Pressure & Volume \\
\hline 1 & 0.9 (Pmna $)$ & 17.681 & 2 & 32.5 & 10.704 \\
1 & 3.0 & 15.855 & 2 & 35.4 & 10.564 \\
1 & 7.3 & 14.603 & 2 & 37.3 & 10.509 \\
1 & 13.8 & 12.866 & 2 & 38.7 & 10.436 \\
2 & 27.3 & 11.367 & 2 & 40.4 & 10.296 \\
2 & 28.0 & 11.104 & 2 & 44.7 & 10.043 \\
2 & 29.3 & 10.985 & & & \\
\hline
\end{tabular}


Table S4. Lattice parameters and volume of the $P 2 / c$ phase as a function of pressure obtained in present theoretical calculations.

\begin{tabular}{cccccc}
\hline $\begin{array}{c}\text { Pressure } \\
\text { (GPa) }\end{array}$ & $\begin{array}{c}\mathbf{a} \\
(\boldsymbol{A})\end{array}$ & $\begin{array}{c}\mathbf{b} \\
(\boldsymbol{A})\end{array}$ & $\begin{array}{c}\mathbf{c} \\
(\boldsymbol{A})\end{array}$ & $\begin{array}{c}\boldsymbol{\beta} \\
\left({ }^{\circ}\right)\end{array}$ & $\begin{array}{c}\mathbf{V} \\
\left(\AA^{3}\right)\end{array}$ \\
\hline 0 & 3.682 & 3.678 & 13.433 & 122.88 & 152.77 \\
2 & 3.532 & 3.532 & 12.209 & 117.77 & 134.76 \\
4 & 3.449 & 3.452 & 11.735 & 116.46 & 125.08 \\
6 & 3.388 & 3.389 & 11.467 & 115.65 & 118.70 \\
10 & 3.298 & 3.297 & 11.080 & 114.56 & 109.57 \\
12.5 & 3.252 & 3.253 & 10.919 & 114.09 & 105.44 \\
20 & 3.149 & 3.152 & 10.611 & 113.09 & 96.85 \\
30 & 3.037 & 3.045 & 10.384 & 111.98 & 89.05 \\
40 & 2.949 & 2.947 & 10.264 & 110.95 & 83.30 \\
50 & 2.876 & 2.875 & 10.187 & 110.23 & 79.00 \\
60 & 2.817 & 2.816 & 10.118 & 109.65 & 75.58 \\
70 & 2.763 & 2.761 & 10.081 & 109.12 & 72.68 \\
80 & 2.722 & 2.719 & 10.019 & 108.74 & 70.22 \\
90 & 2.686 & 2.682 & 9.958 & 108.38 & 68.07 \\
100 & 2.6466 & 2.6455 & 9.938 & 108.06 & 66.16 \\
\hline
\end{tabular}

Table S5. Calculated optical modes of AA-P2/c at $6.0 \mathrm{GPa}$.

\begin{tabular}{cccccccc}
\hline Mode & Symmetry & $\begin{array}{c}\text { Frequency } \\
\text { cm-1 }\end{array}$ & IR & $\begin{array}{c}\text { IR } \\
\text { Intensity }\end{array}$ & Raman & $\begin{array}{c}\text { Raman } \\
\text { Intensity }\end{array}$ & Assignment \\
\hline M4 & $\mathrm{Bg}$ & 112.62 & $\mathrm{~N}$ & 0.00 & $\mathrm{Y}$ & 3.00 & N3 Rot. + NH4 Trans. \\
M5 & $\mathrm{Ag}$ & 133.50 & $\mathrm{~N}$ & 0.00 & $\mathrm{Y}$ & 8.66 & N3 Rot. + NH4 Trans. \\
M6 & $\mathrm{Bg}$ & 280.44 & $\mathrm{~N}$ & 0.00 & $\mathrm{Y}$ & 42.56 & N3 Rot. + NH4 Trans. \\
M7 & $\mathrm{Ag}$ & 284.43 & $\mathrm{~N}$ & 0.00 & $\mathrm{Y}$ & 48.64 & N3 Rot. + NH4 Trans. \\
M8 & $\mathrm{Bg}$ & 325.46 & $\mathrm{~N}$ & 0.00 & $\mathrm{Y}$ & 5.34 & N3 Rot. + NH4 Trans. \\
M9 & $\mathrm{Bg}$ & 186.16 & $\mathrm{~N}$ & 0.00 & $\mathrm{Y}$ & 23.11 & N3 Rot. + NH4 Trans. \\
M10 & $\mathrm{Au}$ & 140.20 & $\mathrm{Y}$ & 3.50 & $\mathrm{~N}$ & 0.00 & Lattice Trans. \\
M11 & $\mathrm{Bu}$ & 140.66 & $\mathrm{Y}$ & 3.71 & $\mathrm{~N}$ & 0.00 & Lattice Trans. \\
M12 & $\mathrm{Bu}$ & 250.06 & $\mathrm{Y}$ & 266.69 & $\mathrm{~N}$ & 0.00 & Lattice Trans. \\
M13 & $\mathrm{Au}$ & 251.38 & $\mathrm{Y}$ & 280.31 & $\mathrm{~N}$ & 0.00 & Lattice Trans. \\
M14 & $\mathrm{Bu}$ & 273.22 & $\mathrm{Y}$ & 562.22 & $\mathrm{~N}$ & 0.00 & Lattice Trans. \\
M15 & $\mathrm{Ag}$ & 218.80 & $\mathrm{~N}$ & 0.00 & $\mathrm{Y}$ & 57.34 & Lattice Rot.
\end{tabular}




\begin{tabular}{|c|c|c|c|c|c|c|c|}
\hline M16 & $\mathrm{Au}$ & 204.11 & $\mathrm{Y}$ & 3.07 & $\mathrm{~N}$ & 0.00 & N3 Trans. \\
\hline M17 & $\mathrm{Bu}$ & 541.82 & $\mathrm{Y}$ & 8.00 & $\mathrm{~N}$ & 0.00 & NH4 Torsion. \\
\hline M18 & $\mathrm{Au}$ & 544.79 & $\mathrm{Y}$ & 3.59 & $\mathrm{~N}$ & 0.00 & NH4 Torsion. \\
\hline M19 & $\mathrm{Bg}$ & 555.10 & $\mathrm{~N}$ & 0.00 & $\mathrm{Y}$ & 0.00 & NH4 Torsion. \\
\hline M20 & $\mathrm{Ag}$ & 556.53 & $\mathrm{~N}$ & 0.00 & $\mathrm{Y}$ & 0.20 & NH4 Torsion. \\
\hline M21 & $\mathrm{Bu}$ & 591.55 & $\mathrm{Y}$ & 9.59 & $\mathrm{~N}$ & 0.00 & NH4 Torsion. \\
\hline M22 & $\mathrm{Bg}$ & 614.70 & $\mathrm{~N}$ & 0.00 & $\mathrm{Y}$ & 0.53 & NH4 Torsion. \\
\hline M23 & $\mathrm{Bu}$ & 617.30 & $\mathrm{Y}$ & 13.79 & $\mathrm{~N}$ & 0.00 & N3 Bend. \\
\hline M24 & $\mathrm{Au}$ & 618.22 & $\mathrm{Y}$ & 11.31 & $\mathrm{~N}$ & 0.00 & N3 Bend. \\
\hline M25 & $\mathrm{Au}$ & 646.16 & $\mathrm{Y}$ & 29.05 & $\mathrm{~N}$ & 0.00 & N3 Bend+NH4 Torsion. \\
\hline M26 & $\mathrm{Bu}$ & 662.68 & $\mathrm{Y}$ & 18.86 & $\mathrm{~N}$ & 0.00 & N3 Bend+NH4 Torsion. \\
\hline M27 & $\mathrm{Bg}$ & 1347.88 & $\mathrm{~N}$ & 0.00 & $\mathrm{Y}$ & 14.16 & $\mathrm{~N}=\mathrm{N}=\mathrm{N}$ Symm str. \\
\hline M28 & $\mathrm{Ag}$ & 1348.76 & $\mathrm{~N}$ & 0.00 & $\mathrm{Y}$ & 479.78 & $\mathrm{~N}=\mathrm{N}=\mathrm{N}$ Symm str. \\
\hline M29 & $\mathrm{Bu}$ & 1422.72 & $\mathrm{Y}$ & 313.49 & $\mathrm{~N}$ & 0.00 & N-H Wagg. + Rock. \\
\hline M30 & $\mathrm{Au}$ & 1423.80 & $\mathrm{Y}$ & 271.67 & $\mathrm{~N}$ & 0.00 & N-H Wagg. + Rock. \\
\hline M31 & $\mathrm{Bu}$ & 1425.94 & $\mathrm{Y}$ & 363.22 & $\mathrm{~N}$ & 0.00 & N-H Wagg. + Rock. \\
\hline M32 & $\mathrm{Bg}$ & 1423.84 & $\mathrm{~N}$ & 0.00 & $\mathrm{Y}$ & 3.98 & N-H Scissor. + Wagg. \\
\hline M33 & $\mathrm{Ag}$ & 1424.49 & $\mathrm{~N}$ & 0.00 & $\mathrm{Y}$ & 12.80 & N-H Scissor. + Wagg. \\
\hline M34 & $\mathrm{Bg}$ & 1439.12 & $\mathrm{~N}$ & 0.00 & $\mathrm{Y}$ & 3.31 & N-H Scissor. + Wagg. \\
\hline M35 & $\mathrm{Au}$ & 1720.20 & $\mathrm{Y}$ & 11.23 & $\mathrm{~N}$ & 0.00 & N-H Bend. \\
\hline M36 & $\mathrm{Ag}$ & 1724.00 & $\mathrm{~N}$ & 0.00 & $\mathrm{Y}$ & 27.50 & N-H Bend. \\
\hline M37 & $\mathrm{Ag}$ & 1752.40 & $\mathrm{~N}$ & 0.00 & $\mathrm{Y}$ & 5.33 & N-H Bend. \\
\hline M38 & $\mathrm{Au}$ & 1744.99 & $\mathrm{Y}$ & 2.37 & $\mathrm{~N}$ & 0.00 & N-H Scissor. + Bend. \\
\hline M39 & $\mathrm{Bu}$ & 2072.51 & $\mathrm{Y}$ & 4960.00 & $\mathrm{~N}$ & 0.00 & $\mathrm{~N}=\mathrm{N}=\mathrm{N}$ Asymm. str. \\
\hline M40 & $\mathrm{Au}$ & 2173.24 & $\mathrm{Y}$ & 211.38 & $\mathrm{~N}$ & 0.00 & $\mathrm{~N}=\mathrm{N}=\mathrm{N}$ Asymm. str. \\
\hline M41 & $\mathrm{Bu}$ & 3011.03 & $\mathrm{Y}$ & 3220.00 & $\mathrm{~N}$ & 0.00 & N-H Asymm. Str. \\
\hline M42 & $\mathrm{Bu}$ & 3022.85 & $\mathrm{Y}$ & 5890.00 & $\mathrm{~N}$ & 0.00 & N-H Asymm. Str. \\
\hline M43 & $\mathrm{Au}$ & 3013.03 & $\mathrm{Y}$ & 2760.00 & $\mathrm{~N}$ & 0.00 & N-H Symm. Str. \\
\hline M44 & $\mathrm{Ag}$ & 3016.87 & $\mathrm{~N}$ & 0.00 & $\mathrm{Y}$ & 2300.00 & N-H Symm. Str. \\
\hline M45 & $\mathrm{Bg}$ & 3017.39 & $\mathrm{~N}$ & 0.00 & $\mathrm{Y}$ & 2290.00 & N-H ASymm. Str. \\
\hline M46 & $\mathrm{Au}$ & 3023.12 & $\mathrm{Y}$ & 232.23 & $\mathrm{~N}$ & 0.00 & N-H Symm. Str. \\
\hline M47 & $\mathrm{Ag}$ & 3027.71 & $\mathrm{~N}$ & 0.00 & $\mathrm{Y}$ & 5148.64 & N-H Symm. Str. \\
\hline M48 & $\mathrm{Bg}$ & 3143.54 & $\mathrm{~N}$ & 0.00 & $\mathrm{Y}$ & 735.46 & N-H ASymm. Str. \\
\hline
\end{tabular}

\section{REFERENCE}

(1) Mills, R.; Olinger, B.; Cromer, D., Structures and Phase Diagrams of $\mathrm{N}_{2}$ and CO to 13 GPa by X-Ray Diffraction. J. Chem. Phys. 1986, 84, 2837-2845.

(2) Yedukondalu, N.; Ghule, V. D.; Vaitheeswaran, G., Computational Study of Structural, Electronic, and Optical Properties of Crystalline $\mathrm{NH}_{4} \mathrm{~N}_{3}$. J. Phys. Chem. C 2012, 116, 16910-16917.

(3) Liu, Q.-J.; Zhang, N.-C.; Wu, J.; Sun, Y.-Y.; Zhang, M.-J.; Liu, F.-S.; Wang, H.-Y.; Liu, Z.-T., Theoretical Insight into the Structural, Elastic and Electronic Properties of $\mathrm{N}_{4} \mathrm{H}_{4}$ 
Compounds. Comput. Mater. Sci. 2014, 81, 582-586.

(4) Liu, Q.-J.; Zeng, W.; Liu, F.-S.; Liu, Z.-T., First-Principles Study of Hydronitrogen Compounds: Molecular Crystalline $\mathrm{NH}_{4} \mathrm{~N}_{3}$ and $\mathrm{N}_{2} \mathrm{H}_{5} \mathrm{~N}_{3}$. Comput. Theor. Chem. 2013, 1014, 37-42.

(5) Yedukondalu, N.; Vaitheeswaran, G.; Anees, P.; Valsakumar, M. C., Phase Stability and Lattice Dynamics of Ammonium Azide under Hydrostatic Compression. Phys. Chem. Chem. Phys. 2015, 17, 29210-29225.

(6) Yu, H.; Duan, D.; Tian, F.; Liu, H.; Li, D.; Huang, X.; Liu, Y.; Liu, B.; Cui, T., Polymerization of Nitrogen in Ammonium Azide at High Pressures. J. Phys. Chem. C 2015, 119, 25268-25272.

(7) Hu, A.; Zhang, F., A Hydronitrogen Solid: High Pressure Ab Initio Evolutionary Structure Searches. J. Phys. Condens. Matter 2010, 23, 022203.

(8) Liu, Q.-J.; Zhang, N.-C.; Sun, Y.-Y.; Zhang, M.-J.; Liu, F.-S.; Liu, Z.-T., DensityFunctional Theory Study of the Pressure-Induced Phase Transition in Hydronitrogen Compound $\mathrm{N}_{4} \mathrm{H}_{4}$. Phys. Lett. A 2014, 378, 1333-1335. 